OPEN ACCESS

Edited by:

Winfried Mayr,

Medical University of Vienna, Austria

Reviewed by:

Fabricio Lima Brasil,

Santos Dumont Institute (ISD), Brazil

Danilo Pani,

University of Cagliari, Italy

*Correspondence:

Guihuai Wang

guihuai_wang@163.com

James Jin Wang

wja01068@btch.edu.cn

Luming $\mathrm{Li}$

lilm@mail.tsinghua.edu.cn

${ }^{\dagger}$ These authors have contributed equally to this work

Specialty section: This article was submitted to Neuroprosthetics,

a section of the journal

Frontiers in Neuroscience

Received: 10 September 2020 Accepted: 16 November 2020

Published: 08 December 2020

Citation:

Lu Y, Xie D, Zhang X, Dong S, Zhang H, Yu B, Wang G, Wang JJ

and Li L (2020) Management of Intractable Pain in Patients With Implanted Spinal Cord Stimulation

Devices During the COVID-19

Pandemic Using a Remote and Wireless Programming System.

Front. Neurosci. 14:594696.

doi: 10.3389/fnins.2020.594696

\title{
Management of Intractable Pain in Patients With Implanted Spinal Cord Stimulation Devices During the COVID-19 Pandemic Using a Remote and Wireless Programming System
}

Yang Lu ${ }^{1,2+}$, Duo Xie ${ }^{2 \dagger}$, Xiaolei Zhang ${ }^{1}$, Sheng Dong ${ }^{1}$, Huifang Zhang ${ }^{1}$, Beibei Yu', Guihuai Wang ${ }^{1 *}$, James Jin Wang ${ }^{1 *}$ and Luming $\mathrm{Li}^{2,3,4,5 *}$

\footnotetext{
${ }^{1}$ Department of Neurosurgery, Beijing Tsinghua Changgung Hospital, School of Clinical Medicine, Tsinghua University, Beijing, China, ${ }^{2}$ National Engineering Laboratory for Neuromodulation, School of Aerospace Engineering, Tsinghua University, Beijing, China, ${ }^{3}$ Precision Medicine and Healthcare Research Center, Tsinghua-Berkeley Shenzhen Institute, Tsinghua University, Shenzhen, China, ${ }^{4}$ IDG/McGovern Institute for Brain Research at Tsinghua University, Beijing, China, ${ }^{5}$ Institute of Epilepsy, Beijing Institute for Brain Disorders, Beijing, China
}

As COVID-19 rampages throughout the world and has a major impact on the healthcare system, non-emergency medical procedures have nearly come to a halt due to appropriate resource reallocation. However, pain never stops, particularly for patients with chronic intractable pain and implanted spinal cord stimulation (SCS) devices. The isolation required to fight this pandemic makes it impossible for such patients to adjust the parameters or configuration of the device on site. Although telemedicine has shown a great effect in many healthcare scenarios, there have been fewer applications of such technology focusing on the interaction with implanted devices. Here, we introduce the first remote and wireless programming system that enables healthcare providers to perform video-based real-time programming and palliative medicine for pain patients with a SCS implant. During the COVID-19 pandemic from January 23, 2020, the date of lockdown of Wuhan, to April 30, 2020, 34 sessions of remote programming were conducted with 16 patients. Thirteen of the 16 patients required programming for parameter optimization. Improvement was achieved with programming adjustment in 12 of $13(92.3 \%)$ cases. Eleven of the 16 (68.8\%) patients reported that the system was user-friendly and met their needs. Five patients complained of an unstable connection resulting from the low network speed initially, and three of these patients solved this problem. In summary, we demonstrated that a remote wireless programming system can deliver safe and effective programming operations of implantable SCS device, thereby providing palliative care of value to the most vulnerable chronic pain patients during a pandemic.

Clinical Trial Registration: www.clinicaltrials.gov, identifier NCT 03858790. 


\section{INTRODUCTION}

The world has changed rapidly due to the disastrous Coronavirus Disease 2019 (COVID-19) pandemic. By the end of July 2020, there were over 19 million confirmed cases of infection and 712,000 deaths due to COVID-19 worldwide. Many countries have issued strict isolation measures or have at least advocated "social distancing" in people's daily lives. Governments and medical providers have had to reallocate labor and material resources to deal with this abrupt emergency. Thus, elective operations/therapies and non-emergency medical procedures have nearly all come to a halt in most medical centers. Patients are also avoiding visiting medical facilities that may be considered a potential source of infection (Keesara et al., 2020). Consequently, some medical needs could be delayed and not met. For patients with demands that cannot be delayed and are impossible to fulfill with the medical resources on site because of isolation protocols or resource limitations, telemedicine has offered an alternative solution (Keesara et al., 2020).

Telemedicine has gradually been adopted by patients over the last decade (Adamse et al., 2018). During the current COVID-19 pandemic, the demand for treatment at a distance from healthcare providers surged essentially overnight (Calton et al., 2020; Humphreys et al., 2020). These changed conditions have boosted reform of the healthcare system. In response, the Chinese government launched a series of policies to support the development of telemedicine and "cloud hospitals." In March 2020, the United States government announced that telemedicine services would be reimbursed by the Medicare and Drug Enforcement Administration, who allowed the prescription of controlled medicine via telemedicine (ATA, 2020).

In most pain management settings, telemedicine has been used in the form of video consultation, which mainly focuses on complaints that can be orally solved, such as palliative care and self-management skills. Some reviews have identified small to moderate reductions in pain, disability, and psychological symptoms in intervention groups applying telemedicine when compared with the control (including standard pain care or waitlist control) (Buhrman et al., 2016; Mariano et al., 2019). However, no therapeutic difference was found when compared to the active control (such as on-site therapies) (Mariano et al., 2019). Remotely delivered physical exercise interventions have also been proposed to reduce pain in patients as a substitute for the usual care (Adamse et al., 2018). However, for addressing more specific demands in a complex situation such as for a patient with chronic intractable pain who has a spinal cord stimulation (SCS) implant, video-based consultation alone is not sufficient. These patients usually require several programming sessions (mostly over 3 months) to achieve a stable therapeutic effect after implantation. When people with chronic pain are deprived of appropriate assessment and treatment, their condition can worsen significantly. Lead migration, scarrelated impedance change, and habituation to the therapy require further programming and regular adjustments (North et al., 2005; Kumar et al., 2008). Considering that patients with chronic pain usually suffer from depression and anxiety, further negative emotions brought on by COVID-19 could exacerbate the pain (Eccleston et al., 2020). These unmet demands require multi-dimensional interactions and teleprogramming of the stimulator by distance.

Here, we introduce the first time remote and wireless programming system for a SCS implant, which enables chronic pain patients to receive timely adjustment of parameters and configurations with only a smartphone and access to a 4G/5G/WiFi network at home.

\section{MATERIALS AND METHODS}

\section{Participants}

The patients in this study were recruited for a multi-center clinical trial (Lu et al., 2020). Patients with chronic intractable pain were recruited at our center from January 2019 to December 2019. Patients were screened from electronic medical records and then approached to complete the verification of the inclusion/exclusion criteria and provided consent. The inclusion criteria were the following: (1) chronic intractable pain that has lasted for at least 3 months and is refractory to the conservative therapies, including oral medications, nerve block, epidural corticosteroids, physical and psychological rehabilitation therapy, and chiropractic care; (2) aged over 18; (3) good compliance and ability to complete post-operative follow-up; and (4) understanding of the method and willingness to sign the informed consent. The exclusion criteria were the following: (1) pregnancy, breast feeding, plan to be pregnant or unwilling to use contraceptive methods; (2) bleeding complications or coagulation disorders; (3) severe mental or cognitive disorders, leading to inability to cooperate during surgery and postoperative programming; (4) life expectancy of less than oneyear; (5) need for therapy or examination that could not be provided with an implanted pulse generator (IPG), such as magnetic resonance imaging and thermo-therapy; and (6) other inappropriate situations that were determined by the investigators.

All patients were implanted with IPG successfully, including 10 males and six females, with an average age of $60.1 \pm 9.9$ years (range 39-74 years old). None of the patients had a device infection except for one patient who complained of red skin at the IPG incision site but had no fever or pain. Two female patients had mild discomfort at the IPG site, but no further surgical treatment was required. There were three cases of spinal cord injury, two cases of peripheral nerve injury, two cases of brachial plexus avulsion, two cases of failed back surgery syndrome, and one case each of complex regional pain syndrome, neuropathic pain, amputated limb pain, phantom limb pain, failed cervical surgery syndrome, syringomyelia, and lower limb ischemic pain in this study.

\section{Intervention}

In accordance with standard clinical practice (Kapural et al., 2015), patients first underwent a screening phase of SCS (with a percutaneous lead or paddle lead) lasting up to 10-14 days with an external stimulator to determine the short-term response. Patients that experienced $50 \%$ or greater pain reduction based on the visual analog scale from baseline were then eligible to proceed to permanent implantation. 
Stimulation parameters were adjusted to optimally overlap paresthesia with the region of the pain area when the patients left the hospital (mean \pm SD of the minimum and maximum programmed parameters: frequency, $70.2 \pm 30.0 \mathrm{~Hz}$; amplitude, $4.6 \pm 2.8 \mathrm{~mA}$; and pulse width, $310 \pm 148 \mu \mathrm{s}$ ). Subsequently, intraoperative paresthesia testing and associated device programming were performed in the hospital, as needed based on patient feedback in standard clinic visits. Oral analgesics were stabilized from 28 days before enrollment until activation of the implanted SCS system, in addition to perioperative analgesics. Adjustments were then allowed under the guidance of the attending physician as medically necessary.

\section{Introduction of the Remote Programming System}

Based on our previously developed telemedicine system (Chen et al., 2014, 2015c), the SCS remote programming system, called the PINS remote programming system, was developed in 2019. The PINS remote programming system can provide multi-dimensional interaction between healthcare providers and chronic pain patients through a computer with PINS remote programming software installed on the physician side, a smartphone with the PINS remote programming application and patient's external programmer on the IPG patient side, and a 4G/5G/WiFi network with an upload speed of no less than $1 \mathrm{MB}$.

Before the connection, the smartphone and the external programmer-activated IPG were paired. Then, according to the patients' requests or previously stated reservation, the healthcare provider logged into the programming system and established the connection with the patient's smartphone after activation of the IPG with patient's external programmer. The smartphone could receive bidirectional, high-definition, and real-time video signals and programming instructions using the public network. The connections between the smartphone and IPG are generated via Bluetooth. Finally, the IPG could work with the physician's instructions (Figure 1A).

To introduce briefly, the remote programming system consists of three modules: the patient management module, teleprogram module, and video communication module. More details can be found in our patents (Chen et al., 2015a,b,d).

The patient management module was designed to build a stable connection between patients and physicians. A list of the patients and their status is shown on the webpage of the patient management module. The physician could only have access to his or her own patients. Once a patient logs into the system. They send their connection request to the physician. The physician could then choose to accept the request and establish a logical connection with the patient. At the same time, the management module would restrict the physician's permission to connect with other patients.

The teleprogram module was designed to provide a platform for SCS remote programming. After a patient is connected to the physician, the patient listens to programming instructions from the server station, which were sent by the physician from the teleprogram module. A telemetry command will then be sent before the programming begins to ensure that all of the hardware or information links are in place. The physician's computer client could store the patient's medical records, electrode images, and previous programming records (Figure 1B). Healthcare providers can easily implement the adjustments of stimulation parameters (including contact mapping, selection of frequency, amplitude, pulse width, etc.), battery status check, electrode impedance check, and device troubleshooting on the teleprogram module. The programming records of every session will be stored in the physician's client, uploaded to the database, and sent back to patients.

A video-recording function was developed based on the Web Real-Time Communication (WebRTC) technology. An opensource project of the STUN protocol was built to support the main signaling service, including taking advantage of the TURN protocol to penetrate firewalls if direct P2P connectivity cannot be established.

Furthermore, the patient's client was designed as a smartphone terminal aimed at assisting patients in interaction with physicians, symptom management, and setting new adjustment parameters. With this client, patient could choose a physician and make a reservation for the remote programming service at home. Medical history, daily outcome reports, and uploaded imaging examinations of the patients are helpful in the management of symptoms and communication with physicians. As for the patients, the only potential restriction is an unstable network. The smartphone app will initially test the network and pair the programmer, and it then alerts the patients if an unstable connection occurs or pairing fails.

\section{Technological Considerations}

The top two concerns of the remote programming are safety and confidentiality. Several appropriate data transmission protection technologies were adopted to ensure the security and confidentiality of communication, such as Hypertext Transfer Protocol Secure (HTTPS) between server and clients, bidirectional identity authentication technology based on digital certificate and short-distance wireless transmission with Bluetooth data transmission technology. The telemedicine system integrates multi-level data isolation and validation procedures without losing real-time performance. Transmission of programming instructions is encrypted between the mutual identity authenticated servers and clients. Also, safety protection mechanisms include functions of automatic instruction back-up, offline parameter recovery, and emergency stimulator shutdown. All of them are available and can be controlled by either programmer or patient. Furthermore, the notes from each encounter can be uploaded to a cloud-based database and can be accessed by any practitioner from any specialty at any time.

However, accidental disconnection resulted from unstable network, or inappropriate amplitude selection may cause severe adverse event. Unlike face-to-face interaction in hospital in which physicians could solve the problem immediately, we suggest at least one caregiver could keep accompany with the patient during the programming process. All patients and at least one caregiver should be made familiar with the remote programming and be able to cooperate with investigators to complete the whole procedure before leaving the hospital. Also, an incremental increase of the amplitude is recommended. In case any discomfort of patient or disconnection happens, the caregiver 


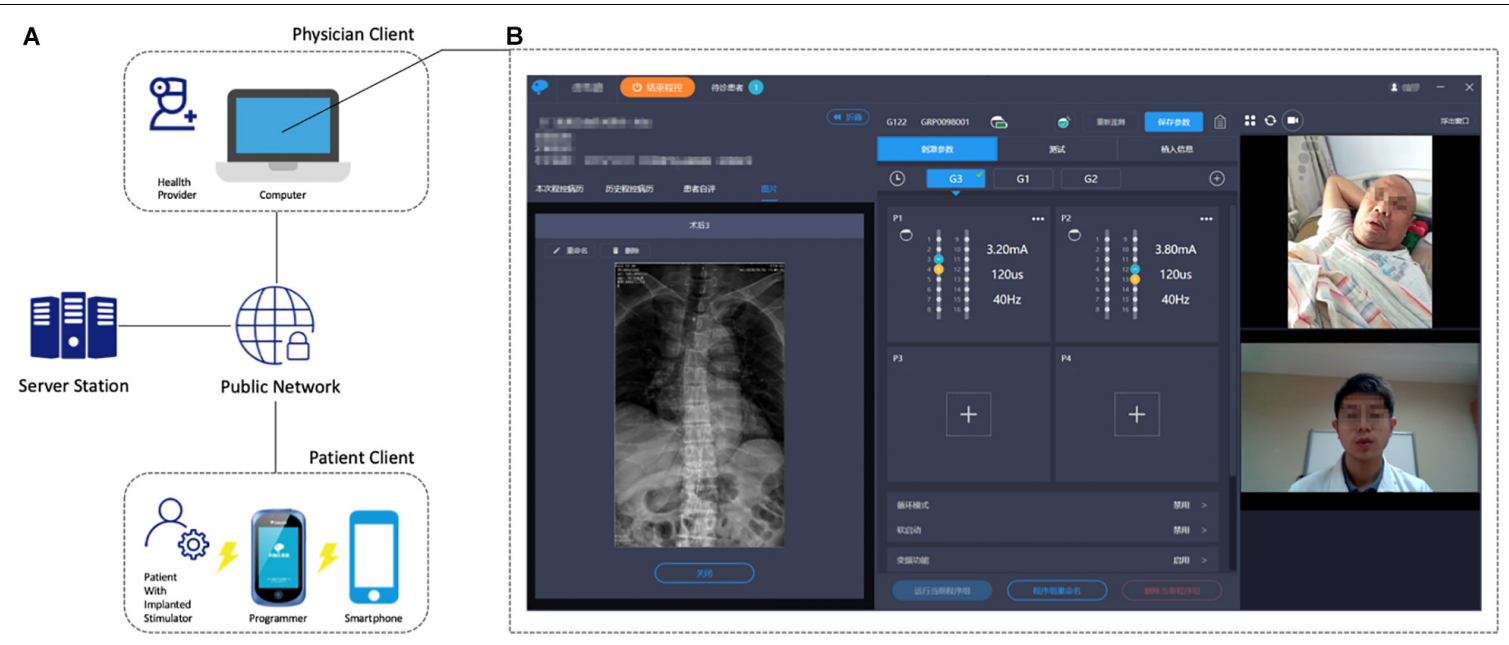

FIGURE 1 | (A) General architecture of the remote programming system. (B) Physician client illustration with the real-time video consultation (right), parameter and configuration adjustment (middle), and the patient's medical record (left). The patient sent a WebRTC request to the signaling server, which is subsequently relayed to the physician client. If an online physician replies to the WebRTC request, a P2P connection will be established, and video stream is facilitated between the two clients.

could restore the pre-programming settings with "one key recovery" or even shutdown the stimulator as quickly as possible.

\section{RESULTS}

The system was launched in 2019, and over 20 patients received this remote-programming-equipped G122R SCS (PINS Medical, Beijing) implantation at our center. From January 23, 2020, the date of lockdown of Wuhan, China, to April 30, 2020, 34 sessions for 16 patients with remote programming were implemented (Table 1). All of the programming practices were performed by a single doctor (Yang Lu) at his home or from our hospital in Beijing. The 16 patients underwent a total of 34 remote programming sessions, with an average adjustment frequency of 2.1 times/patient; the average program control time/adjustment was $43.1 \pm 15 \mathrm{~min}(13-90 \mathrm{~min})$ according to the records of the database.

Our cohort has different types of SCS indications. In contrast to the most common indication for SCS in United States is failed back surgery syndrome (FBSS), spinal-cord-injury-related pain is the most common indication in China. This suggests that patients that undergone more remote programming episodes likely underwent an SCS operation more recently. However, one patient with complex regional pain syndrome completed the remote programming five times owing to the poor quality of the internet connection and device failure (abnormal contact impedance).

Given the thorough penetration of smartphones in China, most of the patients could complete the service on their own or with the assistance of caregivers. Even for the four patients over the age of 70 years who do not use smartphones often, caregivers could help them to proceed with the entire remote programming session.

Thirteen of the 16 patients required programming for parameter optimization. This improvement was achieved with programming adjustment in 12 of 13 cases. Two of the 16 patients sought help because of intolerance to the induced paresthesia with a high amplitude. This is a typical dilemma for the patients using traditional low-frequency stimulation, which requires achieving a balance between the therapeutic effect and paresthesia. The last of the 16 patients experienced reddening of the skin near the incision site but no pain or fever. Since there were no clinical signs of infection, a subsequent follow-up was suggested for monitoring.

Eleven of the 16 patients reported that the system was userfriendly and met their needs. Five patients complained of an unstable connection resulting from a low network speed, three of whom solved this problem by changing the hotspot or using a $4 \mathrm{G}$ network. The other two patients could not solve this problem owing to limited resources. However, these two patients were able to complete the parameter adjustment with our adamant efforts and many reconnection attempts (over $2 \mathrm{~h}$ per patient). No adverse events occurred in the 34 programming sessions.

\section{DISCUSSION}

With regard to neuromodulation therapies, such as SCS, deep brain stimulation (DBS), vagal nerve stimulation, and sacral nerve neuromodulation, implantation of the device is only the first step, and post-operative programming is important for achieving long-term curative effect. Previous studies have proposed that remote programming technology could provide a safe, reliable, and efficient programming service for patients with reduced time and financial cost (Zhang et al., 2019, 2020b). This study represents the first introduction of remote programming technology for patients with chronic pain implanted with an SCS device.

Similar to DBS, patients implanted with an SCS device could receive programming in a face-to-face fashion or adjust the parameters by themselves at home within a limited range preset by the physicians. Self-adjustment has 
proved to be feasible and practical, and it significantly reduces the consultation time (Bally et al., 2019). However, this self-adjustment also needs to be performed under the supervision of the treating physicians, and patients cannot deal with a specific contact change or hardware malfunction by themselves. This had led to a huge demand for a remote programming system for these neuromodulation therapies, especially under extreme conditions such as the current COVID19 pandemic.
For the patients with unsatisfactory post-operative outcomes with traditional parameters or configurations, many novel technologies have been developed in the field of neuromodulation therapies over the last few years, including current-based programming, interleaved programming, fractionated current, variable frequency stimulation, and directional current-steering technologies in DBS (Jia et al., 2017; Wagle Shukla et al., 2017) as well as $10-\mathrm{kHz}$ highfrequency stimulation (Kapural et al., 2015), burst stimulation

TABLE 1 | Characteristics of the 16 patients who received remote programming during the COVID-19 pandemic.

\begin{tabular}{|c|c|c|c|c|c|c|c|c|}
\hline No. & Gender & Age & Diagnosis & Operation date & $\begin{array}{l}\text { Programming } \\
\text { times }\end{array}$ & Reason & Outcome & $\begin{array}{l}\text { Patient's rating for the } \\
\text { remote programming }\end{array}$ \\
\hline 1 & $M$ & 62 & $\begin{array}{l}\text { Periphery nerve } \\
\text { injury }\end{array}$ & $2019 / 11 / 5$ & 3 & $\begin{array}{l}\text { Therapeutic effect } \\
\text { decrease }\end{array}$ & $\begin{array}{l}\text { Parameters adjustment } \\
\text { (little improvement) }\end{array}$ & User-friendly \\
\hline 2 & M & 62 & CRPS & 2019/7/11 & 5 & $\begin{array}{l}\text { Therapeutic effect } \\
\text { decrease (abnormal } \\
\text { impedance of } \\
\text { contacts) }\end{array}$ & $\begin{array}{c}\text { Parameters and } \\
\text { configuration adjustment } \\
\text { (considerate improvement) }\end{array}$ & $\begin{array}{c}\text { Connection unstable } \\
\text { (resolved with changed } \\
\text { network) }\end{array}$ \\
\hline 3 & $\mathrm{~F}$ & 54 & FBSS & 2019/12/25 & 2 & $\begin{array}{l}\text { Therapeutic effect } \\
\text { decrease }\end{array}$ & $\begin{array}{c}\text { Parameters and } \\
\text { configuration adjustment } \\
\text { (considerate improvement) }\end{array}$ & User-friendly \\
\hline 4 & $\mathrm{~F}$ & 55 & Neuropathic pain & 2019/12/24 & 2 & $\begin{array}{l}\text { Therapeutic effect } \\
\text { decrease }\end{array}$ & $\begin{array}{c}\text { Parameters and } \\
\text { configuration adjustment } \\
\text { (considerate improvement) }\end{array}$ & User-friendly \\
\hline 5 & $M$ & 56 & $\begin{array}{l}\text { Amputated limb } \\
\text { pain }\end{array}$ & 2019/12/31 & 3 & $\begin{array}{c}\text { Therapeutic effect } \\
\text { unstable }\end{array}$ & $\begin{array}{l}\text { Change to sub-threshold } \\
\text { stimulation (considerate } \\
\text { improvement) }\end{array}$ & User-friendly \\
\hline 6 & $\mathrm{M}$ & 74 & FBSS & 2019/8/13 & 2 & $\begin{array}{l}\text { Intolerance to the } \\
\text { traditional } \\
\text { stimulation pattern }\end{array}$ & $\begin{array}{l}\text { Oral comforting with lower } \\
\text { amplitude }\end{array}$ & Connection unstable \\
\hline 7 & $M$ & 55 & $\begin{array}{l}\text { Brachial plexus } \\
\text { avulsion }\end{array}$ & $2019 / 5 / 23$ & 1 & $\begin{array}{l}\text { Therapeutic effect } \\
\text { decrease }\end{array}$ & $\begin{array}{c}\text { Parameters adjustment (no } \\
\text { improvement) }\end{array}$ & $\begin{array}{l}\text { Connection unstable } \\
\text { (resolved with changed } \\
\text { network) }\end{array}$ \\
\hline 8 & $M$ & 55 & Phantom limb pain & $2020 / 1 / 3$ & 3 & $\begin{array}{l}\text { Therapeutic effect } \\
\text { decrease }\end{array}$ & $\begin{array}{c}\text { Parameters and } \\
\text { configuration adjustment } \\
\text { (considerate improvement) }\end{array}$ & $\begin{array}{l}\text { Connection unstable } \\
\text { (resolved with changed } \\
\text { network) }\end{array}$ \\
\hline 9 & $\mathrm{~F}$ & 70 & Spinal cord injury & 2019/7/17 & 3 & $\begin{array}{c}\text { Therapeutic effect } \\
\text { unstable }\end{array}$ & $\begin{array}{c}\text { Parameters and } \\
\text { configuration adjustment } \\
\text { (some improvement) }\end{array}$ & User-friendly \\
\hline 10 & $M$ & 74 & Spinal cord injury & $2019 / 7 / 4$ & 1 & $\begin{array}{l}\text { Therapeutic effect } \\
\text { decrease }\end{array}$ & $\begin{array}{l}\text { Parameters adjustment } \\
\text { (little improvement) }\end{array}$ & Connection unstable \\
\hline 11 & $\mathrm{~F}$ & 73 & FCSS & 2019/10/28 & 2 & $\begin{array}{l}\text { Therapeutic effect } \\
\text { unstable }\end{array}$ & $\begin{array}{c}\text { Parameters and } \\
\text { configuration adjustment } \\
\text { (some improvement) }\end{array}$ & User-friendly \\
\hline 12 & $\mathrm{~F}$ & 39 & Spinal cord injury & 2019/8/13 & 1 & $\begin{array}{c}\text { Red incision, } \\
\text { without fever or } \\
\text { pain }\end{array}$ & Continuous follow-up & User-friendly \\
\hline 13 & $M$ & 68 & Syringomyelia & $2019 / 7 / 29$ & 2 & $\begin{array}{l}\text { Therapeutic effect } \\
\text { decrease }\end{array}$ & $\begin{array}{c}\text { Parameters and } \\
\text { configuration adjustment } \\
\text { (some improvement) }\end{array}$ & User-friendly \\
\hline 14 & $M$ & 48 & $\begin{array}{l}\text { Lower limb } \\
\text { ischemic pain }\end{array}$ & 2019/8/6 & 1 & $\begin{array}{l}\text { Therapeutic effect } \\
\text { decrease }\end{array}$ & $\begin{array}{c}\text { Parameters and } \\
\text { configuration adjustment } \\
\text { (some improvement) }\end{array}$ & User-friendly y \\
\hline 15 & $\mathrm{~F}$ & 57 & $\begin{array}{l}\text { Brachial plexus } \\
\text { avulsion }\end{array}$ & 2019/7/17 & 1 & $\begin{array}{l}\text { Intolerance to the } \\
\text { traditional } \\
\text { stimulation pattern }\end{array}$ & $\begin{array}{l}\text { Parameters adjustment } \\
\text { (little improvement) }\end{array}$ & User-friendly \\
\hline 16 & M & 59 & $\begin{array}{c}\text { Periphery nerve } \\
\text { injury }\end{array}$ & 2019/12/9 & 2 & $\begin{array}{l}\text { Therapeutic effect } \\
\text { decrease }\end{array}$ & $\begin{array}{l}\text { Parameters adjustment } \\
\text { (little improvement) }\end{array}$ & User-friendly \\
\hline
\end{tabular}

CRPS, complex regional pain syndrome; FBSS, failed back surgery syndrome; FCSS, failed cervical surgery syndrome. 
(Ahmed et al., 2018), and high-density stimulation in SCS (De Jaeger et al., 2017). These new paradigms require a deep understanding of the underlying therapies. Some stimulation patterns require several hours to several days to achieve a real and optimal response. There is no doubt that a physician's operation and follow-up are inevitable additions to these patterns. Remote programming enables physicians to switch between different stimulation patterns and observe the therapeutic effect efficiently and conveniently.

Several retrospective studies have explored the effect of the DBS remote programming (Zhang et al., 2020a,b), which showed that over $80 \%$ of the patients were satisfied with the remote programming. Evaluation of the patients' symptoms is always a challenge for DBS remote programming. Bradykinesia, tremor, and dyskinesia are easy to follow over video, whereas rigidity is more of a challenge to monitor remotely. In addition, it usually takes longer for the therapeutic effects of DBS to be evident for symptoms such as dyskinesia and tremors. By contrast, patients with pain could receive a real-time response to the stimulation in most cases. In this regard, symptom evaluation is much easier for the SCS remote programming relative to DBS.

Furthemore, pain treatment is not only about pain suppression itself, which has been particularly evident during the COVID-19 pandemic (Cohen et al., 2020) and has also emerged in this study. Within the 34 programming sessions, many patients expressed their anxiety and stress about their situation, especially those who were receiving inadequate care owing to greater isolation due to the lockdown. Anxiety and hopelessness are exacerbated by social isolation, and these negative moods aggravate the pain in a vicious cyclical manner. Thus, it is important to deliver palliative care for these patients in a timely manner. Healthcare providers need to be aware that many of the behavioral components of remote programming are not only potentially helpful for managing pain but also for emotional distress related to the COVID-19 pandemic. A retrospective study on DBS remote programming also found that some patients were satisfied with the programming sessions with no adjustment of the parameters required during the COVID-19 pandemic. This could be due to their expectations and the quality of the psychological support they received during the programming sessions (Zhang et al., 2020a). Empowered by the real-time video-based remote programming system, the demand for management of emotional distress could be met. We believe that reassuring SCS patients that they will be supported and their problems will be addressed during this pandemic at home is as important as the remote programming process.

Remote programming is cost-effective and convenient for patients with SCS and their families. However, before the interaction with healthcare providers can be efficiently established, several preparations need to be in place; these may include measures that ensure patients or their families know to use a smartphone and to keep updating the programming application using a stable network environment. As patients and at least one caregiver are requested to be familiar with the remote programming system, we expect to continue to face obstacles such as communication failure, poor network speed, and difficulty in following the orders when using the smartphone on their first attempt. In addition, a remote programming session is usually more laborious and time-consuming than face-to-face programming. For healthcare providers, patience and practice will help to ensure effective and efficient implementation of remote programming. For technology companies, improving the quality and efficiency is always a goal. We believe that with the continued development of technology, the system could become more intelligent, efficient, and effective at low cost.

If an increasing number of patients access to the remote programming service, the workflow should be designed to minimize the healthcare provider's burden. The teleprogramming team should be organized, including physicians, nurses, and technicians. Team cooperation could schedule visits, remotely train patients and families, resolve technological problems, and optimize the workflow. We suggest that before the start of formal remote programming, team members should recheck the schedule, smooth the network, and ensure patient and family computer literacy.

At the beginning of 2020, the whole world witnessed this unprecedented pandemic; everyone is a fighter against the invisible enemy, and we must all practice "social distancing." During this invisible war, patients with chronic intractable pain with an implanted SCS implant suffer from exacerbated pain helplessly and cannot receive individualized programming on site under these circumstances of isolation. We have demonstrated that the PINS remote wireless programming system allows for connection between patients and healthcare providers within this context, delivers safe and effective remote programming services, and provides palliative care value to the most vulnerable population of chronic pain patients.

With clinical implementation and feedback, we concluded that this remote wireless programming system is an effective and safe method for delivering parameter adjustment, thus improving the quality of life of chronic pain patients.

\section{DATA AVAILABILITY STATEMENT}

The raw data supporting the conclusions of this article will be made available by the authors, without undue reservation.

\section{ETHICS STATEMENT}

The studies involving human participants were reviewed and approved by Ethics committee of Beijing Tsinghua Changgung Hospital. The patients/participants provided their written informed consent to participate in this study. Written informed consent was obtained from the individual(s) for the publication of any potentially identifiable images or data included in this article.

\section{AUTHOR CONTRIBUTIONS}

GW and LL contributed to the conception, the study design, the acquisition, the analysis, the interpretation of data, and the preparation of the manuscript. JW contributed to the study design and the editing of the manuscript. YL contributed to 
the acquisition of data, manuscript writing, and the editing of the manuscript. DX contributed to the study design, the interpretation of data, and editing of the manuscript. SD and $\mathrm{XZ}$ contributed to the study design, the analysis, and the interpretation of data. BY and $\mathrm{HZ}$ contributed to the interpretation of data and the editing of the manuscript. All authors contributed to the article and approved the submitted version.

\section{REFERENCES}

ATA, (2020). Policy Update, 3.17.20. 2020. Available at: https://info. americantelemed.org/covid-19-cms-hhs-dea-updates-3-17-20 (accessed March 22, 2020).

Adamse, C., Dekker-Van Weering, M. G., van Etten-Jamaludin, F. S., and Stuiver, M. M. (2018). The effectiveness of exercise-based telemedicine on pain, physical activity and quality of life in the treatment of chronic pain: a systematic review. J. Telemed. Telecare 24, 511-526. doi: 10.1177/1357633X17716576

Ahmed, S., Yearwood, T., De Ridder, D., and Vanneste, S. (2018). Burst and high frequency stimulation: underlying mechanism of action. Expert. Rev. Med. Devices 15, 61-70. doi: 10.1080/17434440.2018.1418662

Bally, J. F., Rohani, M., Ruiz-Lopez, M., Paramanandam, V., Munhoz, R. P., Hodaie, M., et al. (2019). Patient-adjusted deep-brain stimulation programming is time saving in dystonia patients. J. Neurol. 266, 2423-2429. doi: 10.1007/ s00415-019-09423-9429

Buhrman, M., Gordh, T., and Andersson, G. (2016). Internet interventions for chronic pain including headache: a systematic review. Int. Interv. 4, 17-34. doi: 10.1016/j.invent.2015.12.001

Calton, B., Abedini, N., and Fratkin, M. (2020). Telemedicine in the time of coronavirus. J. Pain Symptom Manag. 60, e12-e14. doi: 10.1016/j.jpainsymman. 2020.03.019

Chen, Y., Chen, H., Ma, B., Hao, H., Li, L., and Beijing Pins Medical. (2015a). A Working Method of Remote Monitoring System for Implanted Medical Equipment. China patent application. CN201510115286.2.

Chen, Y., Chen, H., Ma, B., Lu, C., Hao, H., Li, L., et al. (2015b). Patient Client of Remote Monitoring System for Implanted Medical Equipment. China patent application CN201510114369.X.

Chen, Y., Hao, H., Chen, H., and Li, L. (2015c). The study on a telemedicine interaction mode for deep brain stimulation postoperative follow-up. Conf. Proc. IEEE Eng. Med. Biol. Soc. 2015, 186-189. doi: 10.1109/EMBC.2015. 7318331

Chen, Y., Hao, H., Chen, H., Tian, Y., and Li, L. (2014). The study on a realtime remote monitoring system for Parkinson's disease patients with deep brain stimulators. Conf. Proc. IEEE Eng. Med. Biol. Soc. 2014, 1358-1361. doi: 10.1109/EMBC.2014.6943851

Chen, Y., Hao, H., Li, L., Chen, H., Lu, C., Ma, B., et al. (2015d). A Remote Monitoring System for Implanted Medical Equipment. China patent application CN201510114358.1.

Cohen, S. P., Baber, Z. B., Buvanendran, A., McLean, L., Chen, Y., Hooten, W. M., et al. (2020). Pain management best practices from multispecialty organizations during the COVID-19 pandemic and public health crises. Pain Med. 21, 1331-1346. doi: 10.1093/pm/pnaa127

De Jaeger, M., van Hooff, R. J., Goudman, L., Valenzuela Espinoza, A., Brouns, R., Puylaert, M., et al. (2017). High-density in spinal cord stimulation: virtual expert registry (DISCOVER): study protocol for a prospective observational trial. Anesth. Pain Med. 7:e13640. doi: 10.5812/aapm.13640

Eccleston, C., Blyth, F. M., Dear, B. F., Fisher, E. A., Keefe, F. J., Lynch, M. E., et al. (2020). Managing patients with chronic pain during the COVID-19 outbreak: considerations for the rapid introduction of remotely supported (eHealth) pain management services. Pain 161, 889-893. doi: 10.1097/j.pain. 0000000000001885

Humphreys, J., Schoenherr, L., Elia, G., Saks, N. T., Brown, C., Barbour, S., et al. (2020). Rapid implementation of inpatient telepalliative medicine consultations during COVID-19 Pandemic. J. Pain Symptom Manag. 60, e54-e59. doi: 10. 1016/j.jpainsymman.2020.04.001

\section{FUNDING}

This study was supported by the National Key Research and Development Program of China (2016YFC0105502), National Natural Science Foundation of China (81527901), Shenzhen International Cooperative Research Project (GJHZ20180930110402104), and Beijing Science and Technology Project (Z171100001017109).

Jia, F., Hu, W., Zhang, J., Wagle Shukla, A., Almeida, L., Meng, F. G., et al. (2017). Variable frequency stimulation of subthalamic nucleus in Parkinson's disease: rationale and hypothesis. Park. Relat. Disord. 39, 27-30. doi: 10.1016/j. parkreldis.2017.03.015

Kapural, L., Yu, C., Doust, M. W., Gliner, B. E., Vallejo, R., Sitzman, B. T., et al. (2015). Novel 10-kHz high-frequency therapy (HF10 Therapy) is superior to traditional low-frequency spinal cord stimulation for the treatment of chronic back and leg pain: the senza-rct randomized controlled trial. Anesthesiology 123, 851-860. doi: 10.1097/ALN.0000000000000774

Keesara, S., Jonas, A., and Schulman, K. (2020). Covid-19 and health care's digital revolution. N. Engl. J. Med. 382:e82. doi: 10.1056/NEJMp2005835

Kumar, K., Taylor, R. S., Jacques, L., Eldabe, S., Meglio, M., Molet, J., et al. (2008). The effects of spinal cord stimulation in neuropathic pain are sustained: a 24month follow-up of the prospective randomized controlled multicenter trial of the effectiveness of spinal cord stimulation. Neurosurgery 63, 762-770. doi: 10.1227/01.NEU.0000325731.46702.D9

Lu, Y., Mao, P., Wang, G., Tao, W., Xiong, D., Ma, K., et al. (2020). Spinal cord stimulation for chronic intractable trunk or limb pain: study protocol for a Chinese multicenter randomized withdrawal trial (CITRIP study). Trials 21:834. doi: 10.1186/s13063-020-04768-4763

Mariano, T. Y., Wan, L., Edwards, R. R., and Jamison, R. N. (2019). Online teletherapy for chronic pain: a systematic review. J. Telemed. Telecare 5, 1357633X19871746. doi: 10.1177/1357633X19871746

North, R. B., Kidd, D. H., Farrokhi, F., and Piantadosi, S. A. (2005). Spinal cord stimulation versus repeated lumbosacral spine surgery for chronic pain: a randomized, controlled trial. Neurosurgery 56, 98-106.

Wagle Shukla, A., Zeilman, P., Fernandez, H., Bajwa, J. A., and Mehanna, R. (2017). DBS programming: an evolving approach for patients with Parkinson's disease. Parkinsons Dis. 2017:8492619. doi: 10.1155/2017/8492619

Zhang, C., Zhu, K., Lin, Z., Huang, P., Pan, Y., Sun, B., et al. (2020a). Utility of deep brain stimulation telemedicine for patients with movement disorders during the COVID-19 outbreak in China. Neuromodulation doi: 10.1111/ner.13274

Zhang, J., Hu, W., Chen, H., Meng, F., Li, L., and Okun, M. S. (2020b). Implementation of a novel bluetooth technology for remote deep brain stimulation programming: the pre- and post-covid-19 beijing experience. Mov. Disord. 35, 909-910. doi: 10.1002/mds.28098

Zhang, P., Zhang, Y. G., Liao, L. M., Shen, J. W., Yang, Y. B., Zhang, J. Z., et al. (2019). Application of internet+-based Tsinghua PINS remote tech to improve sacral neuromodulation programming procedure. Int. Urol. Nephrol. 51, 627-632. doi: 10.1007/s11255-019-02115-2113

Conflict of Interest: JW and GW have received research support from Beijing PINS Medical Co., (donated SCS devices for pain). LL reports personal fees from Beijing Pins Medical Co., outside the submitted work.

The remaining authors declare that the research was conducted in the absence of any commercial or financial relationships that could be construed as a potential conflict of interest.

Copyright (c) $2020 \mathrm{Lu}$, Xie, Zhang, Dong, Zhang, Yu, Wang, Wang and Li. This is an open-access article distributed under the terms of the Creative Commons Attribution License (CC BY). The use, distribution or reproduction in other forums is permitted, provided the original author(s) and the copyright owner(s) are credited and that the original publication in this journal is cited, in accordance with accepted academic practice. No use, distribution or reproduction is permitted which does not comply with these terms. 